\title{
Monocyte chemotactic protein-1 in the migration of differentiated leukaemic cells toward alveolar epithelial cells
}

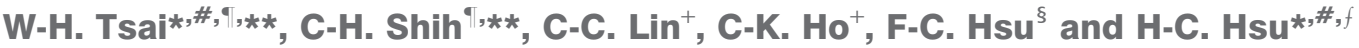

ABSTRACT: All-trans retinoic acid (ATRA) can induce acute respiratory distress syndrome in patients with acute promyelocytic leukaemia (APL). The current study investigated the role of monocyte chemotactic protein (MCP)-1 in the chemotactic transmigration of ATRA-treated NB4 (ATRA-NB4) APL cells toward A549 alveolar epithelial cells.

NB4 and A549 cells were separately cultured with ATRA and/or dexamethasone (DEX). ATRANB4 cells were then placed in an upper insert and co-incubated with A549 cells or their conditioned medium (CM) located in a lower plate to test their transmigration activity.

ATRA stimulated NB4 cells to transmigrate toward the A549 cells. The secretion of MCP-1 was enhanced by ATRA treatment in both A549 and NB4 cells. The binding assay demonstrated that ATRA-NB4 cells bound MCP-1. Pre-treatment of both CM-A549 cells with antibodies against MCP1 and of ATRA-NB4 cells with antibodies against MCP-1 receptors reduced ATRA-NB4 cell transmigration. DEX did not suppress MCP-1 secretion and transmigration in ATRA-NB4 cells, although when applied to A549 cells, MCP-1 secretion was suppressed and ATRA-NB4 cell transmigration was attenuated.

Monocyte chemotactic protein-1 secreted from alveolar epithelial cells plays an important role in the cell-cell interaction involved in the chemotactic transmigration of all-trans retinoic acidtreated acute promyelocytic leukaemia cells toward alveolar epithelial cells.

KEYWORDS: Acute respiratory distress syndrome, all-trans retinoic acid, chemokine, leukocyte infiltration, monocyte chemotactic protein-1, retinoid acid syndrome

A cute promyelocytic leukaemia (APL) is characterised by a translocation $(15 ; 17)$ that leads to an inhibition of terminal granulocytic differentiation [1]. All-trans retinoic acid (ATRA) has been demonstrated to induce differentiation of leukaemia cells into mature granulocytes via a differentiation mechanism [1]. Although this differentiation therapy dramatically improves the outcome of APL, $25-31 \%$ of APL patients develop retinoic acid (RA) syndrome during initial ATRA treatment $[1,2]$. RA syndrome has been shown to have clinical symptoms similar to acute respiratory distress syndrome (ARDS), which include massive infiltration of APL cells found in the alveolar spaces in patients and animals with RA syndrome $[2,3]$. A previous study by TSAI et al. [4], demonstrated that interleukin (IL)-8 and growth-regulated oncogene (GRO)- $\alpha$, secreted from alveolar epithelial cells, play an important role as chemokines for the transmigration of ATRA-treated APL cells toward alveolar epithelial cells. However, pre-treatment with antibodies directly against IL8 and GRO- $\alpha$ receptors does not completely block the transmigration of ATRA treated NB4 cells, therefore suggesting the involvement of other chemokines.

The monocyte chemotactic protein (MCP)-1 (CC chemokine ligand 2: and its receptor CC chemokine receptor 2 (CCR2) are critical determinants for recruitment of leukocytes to inflamed lungs [5-7]. Previous studies have demonstrated that both lung epithelial cells and leukocytes can secret MCP-1 and express its receptors CCR2 [810]. Similarly, ATRA has been reported to increase gene expression or secretion of MCP-1 and CCR2 in APL cells, neutrophils and lung epithelial cells $[8,10,11]$. Therefore, the aim of this study was to investigate the role of MCP-1/ CCR2 axis in the cell-cell interaction essential for the chemotactic transmigration of ATRA-treated NB4 APL cells toward A549 alveolar epithelial cells using a co-culture model.

\section{AFFILIATIONS}

*Dept of Physiology, School of Medicine, National Yang-Ming University,

\#Division of Hematology and Oncology, Dept of Medicine, +Medical Research and Education, Taipei-Veterans General Hospital,

"Dept of Respiratory Therapy, Taipei Medical University,

${ }^{f}$ Dept of Medicine, Taipei City Hospital-Yang-Ming Branch, Taipei, Taiwan, Republic of China. ${ }^{\S}$ Division of Neurology, The Children's Hospital of Philadelphia, Philadelphia, PA, USA.

${ }^{* *}$ Both authors contributed equally to this manuscript.

CORRESPONDENCE

H-C. Hsu

Division of Hematology and Oncology, Dept of Medicine, Taipei City Hospital-Yang-Ming Branch, 105 Yusheng St, Shilin District, Taipei, Taiwan, 11146, Republic of China. Fax: 886228347528

E-mail: hchsu@vghtpe.gov.tw

Received:

October 152007

Accepted after revision:

December 272007

\section{SUPPORT STATEMENT}

This study was supported by grants 93-2314-B-75-024 and 94-2314-B010-013 from the National Science Council, grant VGH 93-C204 from the Taipei-Veterans General Hospital, and a grant from the Taipei City Hospital, Taiwan, Republic of China

\section{STATEMENT OF INTEREST}

None declared. 


\section{MATERIALS AND METHODS}

\section{APL cells and alveolar epithelial cells}

NB4 cells (human APL cell line; a gift from M. Lanotte, INSERM U-301, SDI No 15954.1 CNRS, Centre Hayem, Paris, France ) were cultured in RPMI-1640 medium supplemented with $10 \%$ foetal calf serum (FCS; GIBCO, Grand Island, NY, USA) at a concentration of $1 \times 10^{5}$ cells $\cdot \mathrm{mL}^{-1}$ and incubated at $37^{\circ} \mathrm{C}$ in a humidified incubator with $5 \% \mathrm{CO}_{2}$. The cells were incubated with or without $1 \mu \mathrm{M}$ of ATRA or dexamethasone (DEX) for 3 days before being placed in the upper inserts of the transmigration assay.

The A549 cell (ATCC, Manassas, VA, Canada) is a human alveolar epithelial type II cell line, derived from an individual with alveolar carcinoma. A549 cells retain many characteristics of normal alveolar type II cells and have been extensively used to assess type II alveolar cell function [12]. They were incubated in tissue flasks in a humidified incubator with $5 \% \mathrm{CO}_{2}$ at $37^{\circ} \mathrm{C}$ with Hams' F12K medium (Sigma, St. Louis, MO, USA) supplemented with $10 \%$ FCS, streptomycin $\left(50 \mu \mathrm{g} \cdot \mathrm{mL}^{-1} ; \mathrm{GIBCO}\right)$ and penicillin (50 U.mL $\mathrm{mL}^{-1}$, GIBCO). A549 cells were grown in adhesion as monolayers, cells were then harvested using trypsin and EDTA (0.25 and 0.1\%, respectively; GIBCO) in PBS, centrifuged at low speed $(250 \times g)$ for $5 \mathrm{~min}$ and resuspended in fresh medium at concentration of $4 \times 10^{5} \mathrm{cells} \cdot \mathrm{mL}^{-1}$. Cells were re-incubated on the 24-well plate with or without ATRA $(1 \mu \mathrm{M})$ or DEX $(1 \mu \mathrm{M})$ for $1-3$ days. These cells were also grown in monolayers and used as the lower plate in the co-culture system during the transmigration assay.

\section{Preparation of conditioned medium}

Either NB4 cells $\left(1 \times 10^{5}\right.$ cells $\left.\cdot \mathrm{mL}^{-1}\right)$ or A549 cells $\left(4 \times 10^{5}\right.$ cells $\left.\cdot \mathrm{mL}^{-1}\right)$ were cultured in the flask for $1-3$ days with or without $1 \mu \mathrm{M}$ of ATRA and/or DEX. The supernatants were harvested, centrifuged at $250 \times g$ for $5 \mathrm{~min}$ to remove cellular components and stored as aliquots at $-20^{\circ} \mathrm{C}$.

\section{Chemotaxic cell-transmigration assay}

The chemotaxic cell-transmigration assay was performed in a co-culture system using an upper cell culture insert (BD Falcon $^{\mathrm{TM}}$, San Jose, CA, USA) with polyethylene terephthalate membrane $\left(8 \mu \mathrm{m}\right.$ pore size, $1 \times 10^{5}$ pores $\left.\cdot \mathrm{cm}^{-2}\right)$ and a companion 24-well plate in the bottom (fig. 1). In brief, the NB4 cells pre-treated with or without ATRA were centrifuged at a low speed $(250 \times g, 5 \mathrm{~min})$, re-suspended in fresh medium $\left(1 \times 10^{5}\right.$ cells $\left.\cdot \mathrm{mL}^{-1}\right)$, placed in the upper inserts and incubated with or without antibodies specific for MCP-1 receptors (antiCCR2) and/or IL-8 receptors (anti-CXC chemokine receptor (CXCR)1 and anti-CXCR2; R\&D Systems, Minneapolis, MN, USA) for $2 \mathrm{~h}$ at $37^{\circ} \mathrm{C}$. The lower plate was either pre-coated with an A549 cell monolayer as mentioned previously or filled with various types of conditioned medium (CM), F12K medium alone or F12K medium with defined concentrations of exogenous MCP-1 (fig. 1). The upper insert and lower plate were assembled and incubated in $100 \%$ humidity and $5 \% \mathrm{CO}_{2}$ at $37^{\circ} \mathrm{C}$ for $2 \mathrm{~h}$. After incubation, NB4 cells in the upper insert were removed by scraping. The membrane was then removed from the upper insert, placed on a slide and stained with Wright stain (Merck Co, Taiwan, Republic of China). The number of transmigration of NB4 cells was represented by the sum of the cells transmigrated in the nine fixed fields over each insert membrane under light microscopy at $200 \times$ magnification, as described previously [4].

\section{Assay for chemokine determination}

The levels of MCP-1 in the CM from different cell culture conditions were determined by an ELISA kit following the manufacturer protocols (R\&D Systems).

\section{Statistical analysis}

Data were evaluated by ANOVA test and Wilcoxon signedrank test. A value of $\mathrm{p}<0.05$ was considered significant. All data are presented as mean $\pm \mathrm{SD}$.

\section{RESULTS}

\section{Transmigration of ATRA-treated NB4 cells by A549 cell co- incubation}

After culturing the cells separately for 3 days, ATRA-treated NB4 cells were co-cultured with ATRA-untreated or ATRAtreated A549 cells to determine their transmigration activity. The study by TsAiet al. [4] demonstrated the chemotactic activity in the CM from A549 cell cultures. Co-incubation of ATRAtreated NB4 cells with the CM from either untreated-A549 cells or ATRA-treated A549 cells produced a progressive increase in cell transmigration in a time-dependent manner [4]. Figure 2 shows that ATRA-treated NB4 cells migrate toward untreatedA549 cells and that this cell transmigration was significantly enhanced when A549 cells were pre-treated with ATRA.

\section{Secretion of MCP-1 from A549 and NB4 cells}

The current authors subsequently measured the concentrations of MCP-1 in the various CM obtained from either A549 or NB4 cell cultures. The concentration of MCP-1 in the CM was increased, over time, when untreated-A549 cells (CM-A549) were cultured for 1-3 days (data not shown). ATRA treatment significantly enhanced the secretion of MCP-1 from A549 cells into the CM (CM-A549+ATRA) compared with CM-A549 $(\mathrm{p}<0.005$; fig. 3 ; $)$. In contrast, only a minimal amount of MCP-1 was secreted in the CM over time in untreated-NB4 (CM-NB4) cell cultures. ATRA treatment markedly enhanced the secretion of MCP-1 from NB4 cells into the CM (CMNB4+ATRA, $p<0.0001$; fig. 3).

\section{Role of MCP-1 in the transmigration of ATRA-treated NB4 cells}

To assess the role of MCP-1 in the CM in the transmigration of ATRA-treated NB4 cells, the MCP-1 was neutralised in the CM by pre-treating the CM-A549 cells with anti-MCP-1 antibody

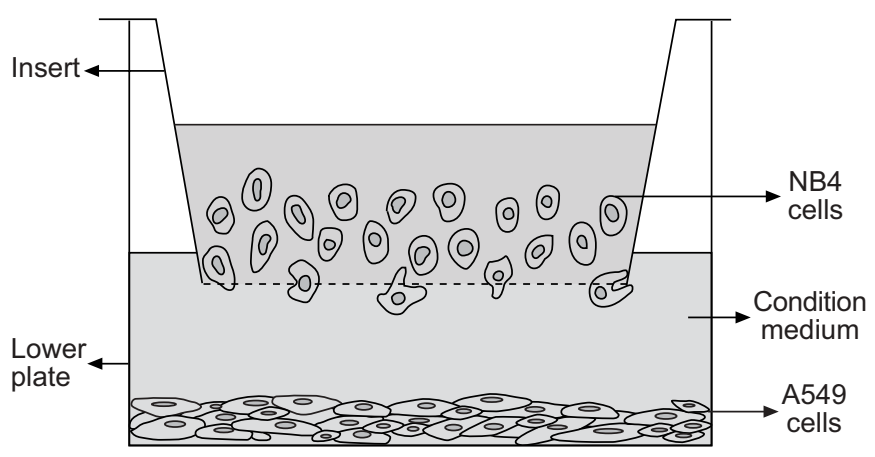

FIGURE 1. Diagram of co-culture and transmigration assay. 


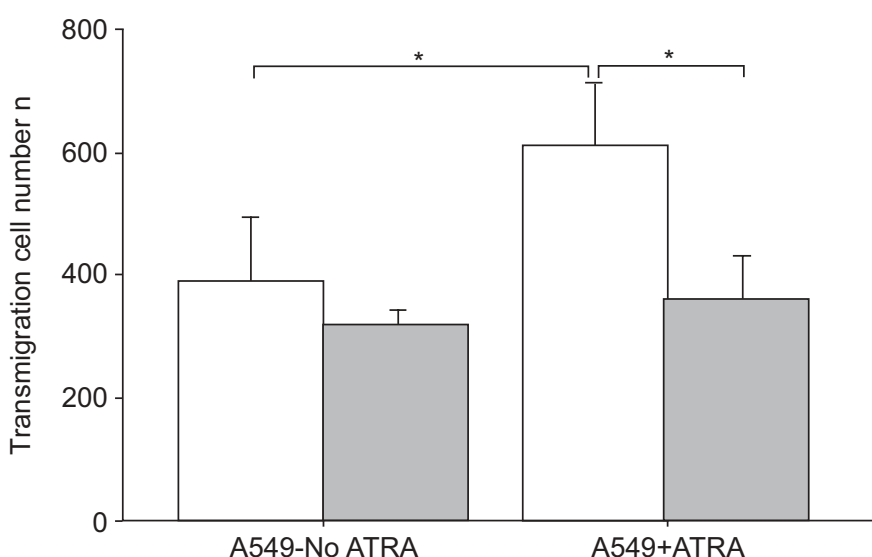

FIGURE 2. Effects of treating A549 cells with all-trans retinoic acid (ATRA) and/ or dexamethasone (DEX) on the transmigration of ATRA-treated NB4 cells. Data are presented as mean \pm SD for five independent experiments. The upper insert was NB4+ATRA. $\square$ : conditioned medium (CM) of A549+DEX; $\square$ : CM of A549 cells. $*: p<0.05$

for $2 \mathrm{~h}$ prior to co-incubation with ATRA-treated NB4 cells. Figure 4 shows that the level of the transmigration of ATRAtreated NB4 cells was reduced when MCP-1 in the CM of A549 cells was neutralised by its specific antibody $(p=0.063)$.

\section{Role of CCR2 in the transmigration of ATRA-treated NB4 cells}

The present authors determined the binding of MCP-1 to both untreated and ATRA-treated NB4 cells after 3 days of culturing. Figure 5 shows that only a minimal amount of MCP-1 binding was detected in untreated-NB4 cells as demonstrated by flow cytometric analysis. Binding of MCP-1 was slightly increased in NB4 cells after treatment with ATRA for 1 day (fig. 5), but was not increased after treatment with ATRA for 3 days.

Subsequently, the CCR2 receptor was blocked on ATRAtreated NB4 cells with a specific antibody to assess its role in the cell transmigration activity. The current authors pre-treated these cells with anti-CCR2 antibody for $2 \mathrm{~h}$ prior to coincubation with the CM of A549 cells. Figure 6 shows that the level of the transmigration of ATRA-treated NB4 cells was reduced when CCR2 was blocked by its specific antibody $(\mathrm{p}<0.05)$.

\section{Effect of DEX on the secretion of MCP-1 from A549 and NB4 cells}

A previous study demonstrated that DEX had no effect on the transmigration of ATRA-treated NB4 cells by itself [4], but can significantly reduced the transmigration of ATRA-treated NB4 cells toward A549 cells when the latter cells were treated with DEX as shown in figure 2.

The present authors further collected the CM from A549 cells or NB4 cells after culturing with or without DEX for 3 days. DEX reduced the secretion of MCP-1 in both untreated- and ATRA-treated A549 cells (fig. 3) although it did not affect the secretion of MCP-1 in both untreated- and ATRA-treated NB4 cells (fig. 3).
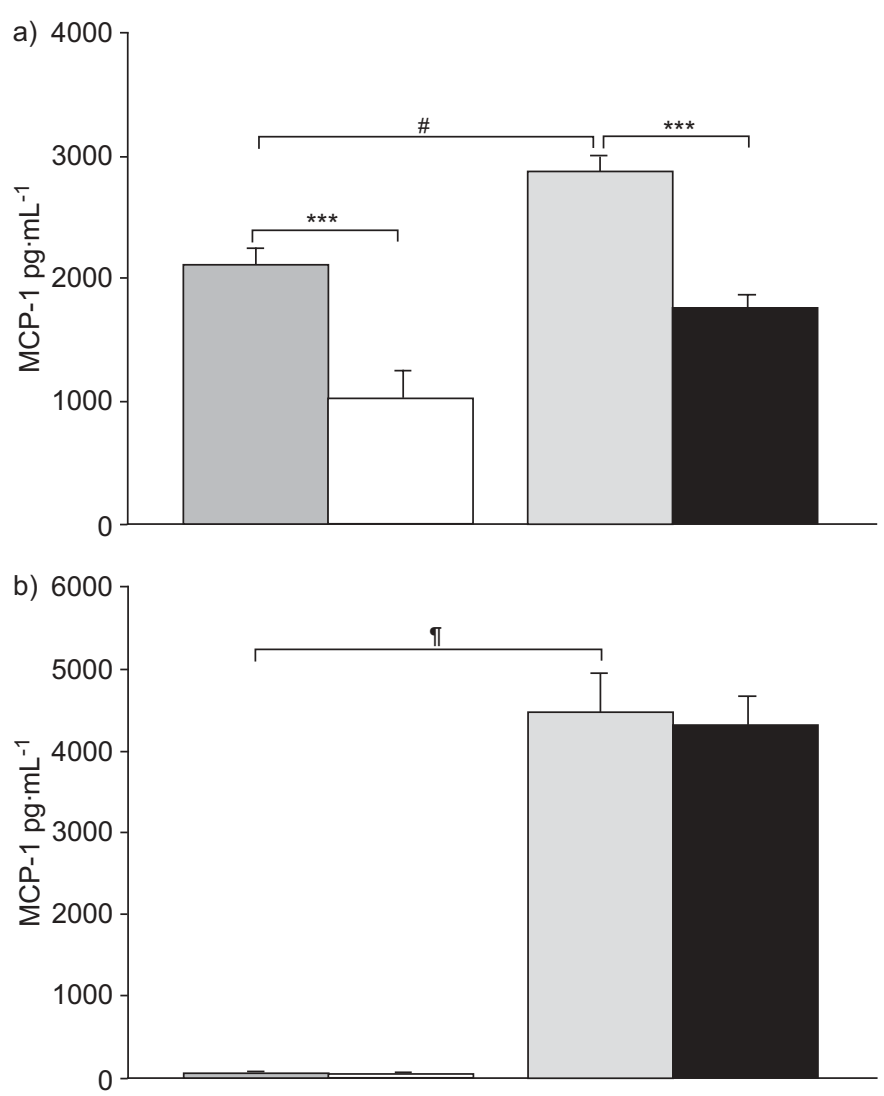

FIGURE 3. Secretion of monocyte chemotactic protein (MCP)-1 by conditioned media in a) A549 cells and b) NB4 cells. Data are presented as mean \pm SD

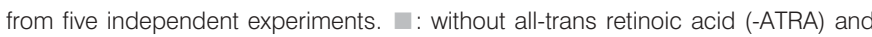
without dexamethasone (DEX); $\square$ : -ATRA+DEX; 1 : +ATRA-DEX; $\mathbf{\square}:+A T R A+D E X$. ${ }^{\#}: p<0.005 ; ": p<0.0001 .{ }^{* * *}: p<0.001$.

\section{Cooperation of MCP-1 and IL-8 in the transmigration of ATRA-treated NB4 cells}

A previous study demonstrated that IL-8 secreted from alveolar epithelial cells is also an important chemokine for the transmigration of ATRA-treated APL cells toward alveolar epithelial cells [4]. To investigate the relative role of both MCP1 and IL- 8 on the ATRA-treated NB4 cell transmigration, the exogenous MCP-1 and IL- 8 were added, either alone or in combination, into the original culture medium and allowed the co-incubation of untreated- or ATRA-treated NB4 cells with these exogenous chemokines for $2 \mathrm{~h}$. The level of transmigration of ATRA-treated NB4 cells was increased when coincubating with either exogenous MCP-1 or IL-8 alone [4], which can be further promoted when the cells were coincubated with both exogenous chemokines at the same time (data not shown). The present authors further confirmed the synergistic effect of MCP-1 and IL-8 in promoting the transmigration activity of ATRA-treated NB4 cells by blocking their surface receptors with specific antibodies. The ATRAtreated NB4 cells were pre-treated with anti-CCR2 (MCP-1 receptor antibody) and/or anti-CXCR1+anti-CXCR2 (IL-8 receptor antibody) for $2 \mathrm{~h}$ prior to co-incubation with the $\mathrm{CM}$ of A549 cells. Figure 6 shows that the level of the transmigration of ATRA-treated NB4 cells was attenuated when either MCP-1 receptors or IL- 8 receptors were blocked by their 


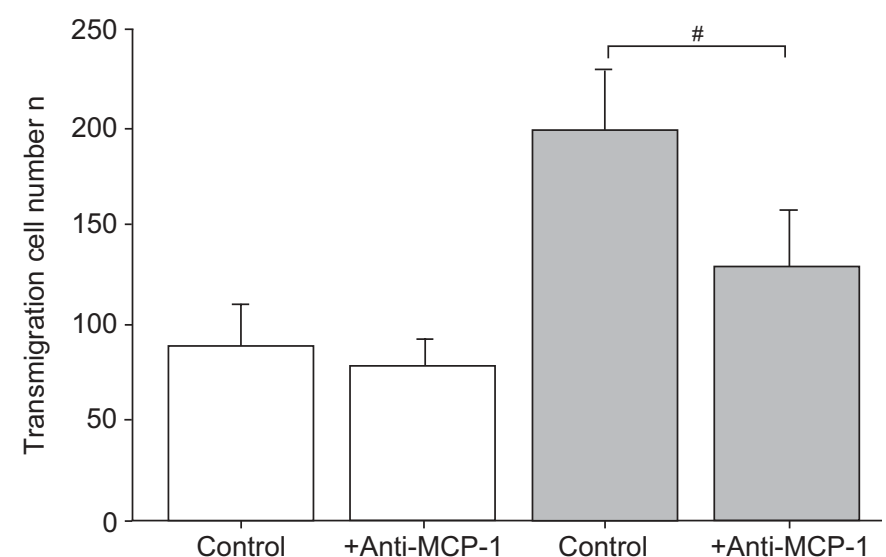

FIGURE 4. Suppression of transmigration of all-trans retinoic acid (ATRA)treated NB4 cells by neutralising monocyte chemotactic protein (MCP)-1. Data are presented as mean \pm SD from six independent experiments. The upper insert was NB4+ATRA. $\square$ : F12K+serum and antibodies; $\square$ : conditioning medium of A549 cells and antibodies. ${ }^{*}: p=0.063$.

specific antibodies $(\mathrm{p}<0.05$ and $\mathrm{p}<0.005$, respectively) [4]. However, the transmigration of ATRA-treated NB4 cells can be further suppressed when receptors of both chemokines were blocked in the same time ( $p<0.01$; fig. 6 ).

\section{DISCUSSION}

The results of the present study demonstrate that ATRA stimulated NB4 cells to transmigrate toward the A549 cells and this promotion of NB4 cell transmigration was mediated through chemoattractants secreted from A549 cells. The results from the current study reveal that MCP-1 secreted from A549 cells was an important chemoattractant to account for the chemotactic transmigration of ATRA-treated NB4 cells. Measurements of the chemokine levels in CM revealed that A549 cells constitutively secrete MCP-1 (fig. 3). The important role of MCP-1 in ATRA-treated NB4 cell transmigration is strongly supported by the findings that: 1) exogenous administration of MCP-1 can bind to the ATRA-treated NB4 cells and increase their transmigration activity; 2) pretreatment of ATRA-treated NB4 cells with antibodies against MCP-1 receptors can largely reduce their transmigration activity; and 3) transmigration of ATRA-treated NB4 cells toward CM of A549 cells was reduced when MCP-1 in the latter $\mathrm{CM}$ was neutralised with its specific antibody (figs 3-6). Collectively, the authors' results suggest that A549 cells have profound chemotactic influences on the promotion of transmigration of ATRA-treated NB4 cells and this cell-cell interaction is mediated through the secretion and function of MCP-1.

In addition the present authors found that untreated-NB4 cells only secrete minimal amounts of MCP-1, and ATRA treatment markedly enhances the secretion of MCP-1 (fig. 3) resulting in an increased difficulty to evaluate the dose effect of exogenous MCP-1 on the ATRA-treated NB4 cells (data not shown). The present study's results are consistent with a previous report that the expression of gene and protein of MCP-1 can be expressed in the ATRA-treated APL cells, as well as in activated neutrophils [8]. Previous studies have reported that
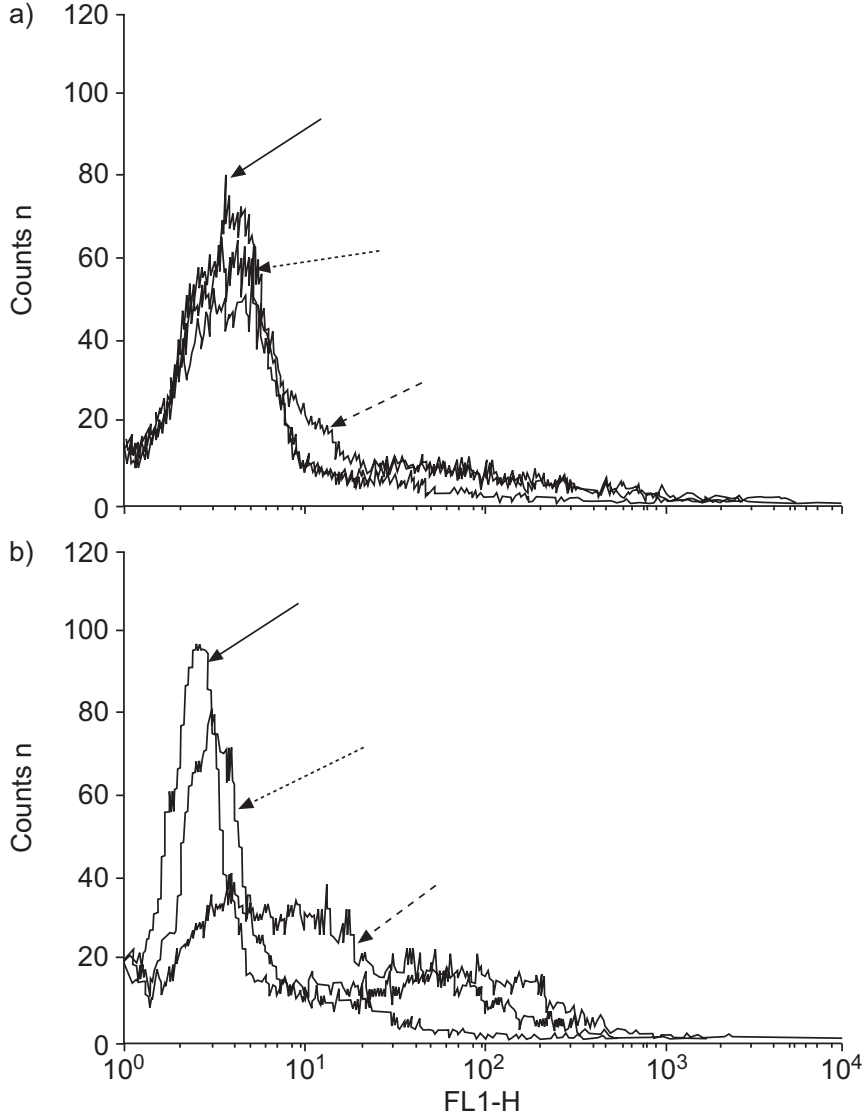

FIGURE 5. Effects of all-trans retinoic acid (ATRA) on the binding of monocyte chemotactic protein (MCP-1) to NB4 cells. a) Untreated and b) ATRA-treated NB4 cells were incubated with biotinylated MCP-1 for $1 \mathrm{~h}$ before analysis with flow cytometry. The specificity of MCP-1 is demonstrated by blocking their binding with specific antibodies. The histogram is representative of four independent experiments. — : control; …... anti-MCP-1; - - -: labelled MCP-1.

binding of MCP-1 to its specific receptors can further downregulate the expression of their surface receptors (CCR2) [13]. This can explain why binding of MCP-1 to NB4 cells was only transiently increased during the early phase of ATRA treatment, but not in later phase (fig. 5). Furthermore, expression of CCR2 is also downregulated by other cytokines secreted by ATRA-treated NB4 cells, such as IL-1 and tumour necrosis factor- $\alpha$ [14]. Accordingly, ATRA treatment in the APL cells induces granulocytic differentiation as well as inducing an underlying mechanism responsible for activation events in the mature neutrophils $[1,2]$. These indicate that MCP-1 plays as an important role for enhancing migration activity in the differentiated APL cells during ATRA treatment.

It has been suggested that chemokines produced by extravascular cells can be transported to the luminal surfaces of the endothelium, where they are presented to leukocytes $[15,16]$. It is also known that MCP-1 can prime and activate leukocytes [8]. Accordingly, MCP-1 secreted from lung epithelial cells, serving as chemotactic mediators, may act on ATRA-treated APL cells and promote their transmigration [10]. Moreover, the increased secretion of MCP-1 and increased binding of CCR2 in the ATRA-treated APL cells function in priming these 


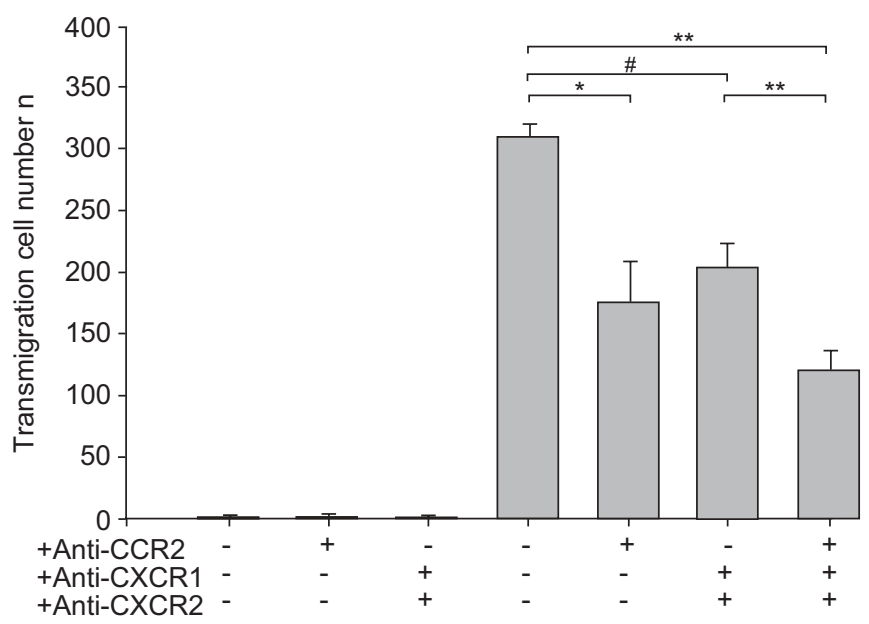

FIGURE 6. Suppression of transmigration of all-trans retinoic acid (ATRA)treated NB4 cells by blocking their surface receptors for monocyte chemotactic protein (MCP)-1 (CC chemokines receptor (CCR) 2) and/or interleukin (IL)-8 (CX chemokine receptor (CXCR) 1 and CXCR2) with specific antibodies. The upper insert was ATRA-treated NB4 cells ( $\square$ ) and NB4 $(\square)$ were previously incubated with or without specific antibodies for MCP-1 receptors (anti-CCR2; $1 \mu \mathrm{g} \cdot \mathrm{mL}^{-1}$ ) and/or IL8 receptors (anti-CXCR1 and anti-CXCR2, both $10 \mu \mathrm{g} \cdot \mathrm{mL}^{-1}$ ) for $2 \mathrm{~h}$ before use Lower plate: conditioned medium of A549 cells that were previously cultured for 3 days. The upper insert and lower plate were then assembled and incubated for $2 \mathrm{~h}$. Data are presented as mean \pm SD taken from seven independent experiments. \#: $p<0.005 ; *: p<0.01 ; * *: p<0.01$.

differentiated APL cells for transmigration mobility [8]. In such a way, MCP-1 produced by both A549 cells and APL cells play an important role in the chemokine network in orchestrating the trafficking of ATRA-treated APL cells to the alveolar spaces in patients with RA syndrome.

DEX has been used successfully to prevent or treat RA syndrome [2]. The current study data demonstrates that when DEX was applied to A549 cells, it suppressed their MCP-1 secretion and attenuated the transmigration of ATRA-treated NB4 cells toward these cells (figs 2 and 3). Conversely, when DEX was applied to ATRA-treated NB4 cells, it had no effect on MCP-1 secretions (fig. 2) and transmigration activity [7]. These data further support the following theories: 1) MCP-1 secreted by alveolar epithelial cells is important in the regulation of transmigration of differentiated APL cells into the alveolar spaces; 2) the therapeutic effect of DEX on the transmigration of ATRA-treated APL cells is actually mediated, in part, through its inhibitory action on MCP-1 secretion in A549 cells, but not in APL cells; and 3) lung epithelial cells are also susceptible to ATRA and DEX treatment. The inhibitory effect of DEX on the MCP-1 secretion from the alveolar epithelial cells has previously been reported [17], and its underlying mechanism is likely to be mediated by glucocorticoid receptor through a novel mechanism in which the glucocorticoid receptor binds directly to MCP-1 mRNA and facilitates its degradation $[18,19]$. However, glucocorticoid binding and cellular glucocorticoid receptor levels were not changed in the APL cells during the process of ATRA-induced granulocytic differentiation [20]. This may explain why DEX has different effects between ATRA-treated A549 cells and ATRA-treated NB4 cells.

Regarding the pathogenesis of RA syndrome, previous investigations have focused on the functional activation of ATRA-treated APL cells for the promotion of the adherence, leukoaggregation, transendothelial migration and the transverse of extracellular matrix [20-25]. It is known that the propagation of inflammatory signals from the airspace to the vascular space is pivotal in lung inflammation [26]. The present authors have adopted a co-culture model to elucidate the chemokine mechanisms underlying the chemotactic transmigration of ATRA-treated APL cells toward alveolar epithelial cells [4]. In addition to IL- 8 and GRO- $\alpha$ reported previously, it has been demonstrated in the current study that MCP-1 also plays an important role in the chemokine network in orchestrating the trafficking of ATRA-treated APL cells to the alveolar space. Furthermore, the study demonstrates that MCP-1 and IL-8 work synergistically in promoting transmigration of ATRA-treated APL cells toward alveolar epithelial cells (fig. 6). This indicates that these two chemokines are interdependent in mediating the transmigration of ATRAtreated APL cells into the alveolar space [4]. In ARDS induced by other pathogens, MCP-1 is an important chemokine during the transition phase from the early-to-late phase of transmigration of leukocytes into the alveolar space [7, 27]. MCP-1 can also induce the transmigration of circulating monocytes into the alveolar space during acute lung injuries [28]. These recruited monocytes can drastically amplify the further transmigration of neutrophils and exacerbate the inflammatory reactions [29]. In animal studies, employing CCR2-knockout mice or blocking CCR2 function by an antibody also demonstrated that suppressed alveolar monocyte recruitment markedly decreased the alveolar neutrophils accumulation, vascular permeability and inflammatory reactions [6]. The amount of circulating normal monocytes is decreased in most APL patients as their normal haematopoiesis is suppressed by the massive leukaemic APL cells in the bone marrow, which can subsequently cause impaired monocyte recruitment into the alveolar spaces in APL patients. The amount of monocytes recruited into alveolar space has not been determined in patients with RA syndrome. Further studies are needed to determine the role of circulating normal monocytes in the development of RA syndrome in APL patients under ATRA treatment.

The present authors conclude that monocyte chemotactic protein-1 secreted from alveolar epithelial cells plays an important role in the cell-cell interaction essential for the chemotactic transmigration of all-trans retinoic acid related acute promyelocytic leukaemia cells toward alveolar epithelial cells. Understanding the mechanism underlying monocyte chemotactic protein- 1 expression and function, therefore, will provide a new target and strategy for therapeutic intervention to treat lung inflammation in retinoic acid syndrome.

\section{REFERENCES}

1 Huang ME, Ye YC, Chen SR, et al. Use of all-trans retinoic acid in the treatment of acute promyelocytic leukemia. Blood 1988; 72: 567-572. 
2 Frankel SR, Eardley A, Lauwers G, Weiss M, Warrell RP Jr. The "retinoic acid syndrome" in acute promyelocytic leukemia. Ann Intern Med 1992; 117: 292-296.

3 Ninomiya M, Kiyoi H, Ito M, Hirose Y, Naoe T. Retinoic acid syndrome in NOD/scid mice induced by injecting an acute promyelocytic leukemia cell line. Leukemia 2004; 18 : 442-448.

4 Tsai WH, Hsu CH, Lin CC, Ho CK, Kou YR. Role of IL-8 and GRO- $\alpha$ in the chemotactic migration of all-trans retinoic acid-treated promyelocytic leukemic cells toward alveolar epithelial cells. Crit Care Med 2007; 35: 879-885.

5 Maus U, Waelsch K, Kuziel WA, et al. Monocytes are potent facilitators of alveolar neutrophil emigration during lung inflammation: role of the CCL2-CCR2 axis. J Immunol 2003; 170: 3273-3278.

6 Maus U, von Grote K, Kuziel WA, et al. The role of CC chemokine receptor 2 in alveolar monocyte and neutrophil immigration in intact mice. Am J Respir Crit Care Med 2002; 166: 268-273.

7 Maus U, Huwe J, Maus R, Seeger W, Lohmeyer J. Alveolar JE/MCP-1 and endotoxin synergize to provoke lung cytokine upregulation, sequential neutrophil and monocyte influx, and vascular leakage in mice. Am J Respir Crit Care Med 2001; 164: 406-411.

8 Burn TC, Petrovick MS, Hohaus S, Rollins BJ, Tenen DG. Monocyte chemoattractant protein-1 gene is expressed in activated neutrophils and retinoic acid-induced human myeloid cell lines. Blood 1994; 84: 2776-27783.

9 Christensen PJ, Du M, Moore B, Morris S, Toews GB, Paine R. Expression and functional implications of CCR2 expression on murine alveolar epithelial cells. Am J Physiol Lung Cell Mol Physiol 2004; 286: 68-72.

10 Thorley AJ, Ford PA, Giembycz MA, Goldstraw P, Young A, Tetley TD. Differential regulation of cytokine release and leukocyte migration by lipopolysaccharidestimulated primary human lung alveolar type II epithelial cells and macrophages. J Immunol 2007; 178: 463-473.

11 Cignetti A, Vallario A, Roato I, et al. The characterisation of chemokine production and chemokine receptor expression reveals possible functional cross-talks in AML blasts with monocytic differentiation. Exp Hematol 2003; 31: 495-503.

12 Baba A, Kim YK, Zhang H, Liu M, Slutsky AS. Perfluorocarbon blocks tumor necrosis factor- $\alpha$-induced interleukin-8 release from alveolar epithelial cells in vitro. Crit Care Med 2000; 28: 1113-1118.

13 Tangirala RK, Murao K, Quehenberger O. Regulation of expression of the human monocyte chemotactic protein-1 receptor (hCCR2) by cytokines. J Biol Chem 1997; 272: 8050-8056.

14 Hsu HC, Tsai WH, Chen PG, Hsu ML, Ho CK, Wang SY. In vitro effect of granulocyte-colony stimulating factor and all-trans retinoic acid on the expression of inflammatory cytokines and adhesion molecules in acute promyelocytic leukemic cells. Eur J Haematol 1999; 63: 11-18.

15 Middleton J, Neil S, Wintle J, et al. Transcytosis and surface presentation of IL-8 by venular endothelial cells. Cell 1997; 91: 385-395.
16 Quinton LJ, Nelson S, Zhang P, et al. Selective transport of cytokine-induced neutrophil chemoattractant from the lung to the blood facilitates pulmonary neutrophil recruitment. Am J Physiol Lung Cell Mol Physiol 2004; 286: L465-L472.

17 Paine R 3rd, Rolfe MW, Standiford TJ, Burdick MD, Rollins BJ, Strieter RM. MCP-1 expression by rat type II alveolar epithelial cells in primary culture. J Immunol 1993; 150: 4561-4570.

18 Dhawan L, Liu B, Blaxall BC, Taubman MB. A novel role for the glucocorticoid receptor in the regulation of monocyte chemoattractant protein-1 mRNA stability. J Biol Chem 2007; 28: 10146-10152.

19 Croxtall JD, van Hal PTW, Choudhury Q, Gilroy DW, Flower RJ. Different glucocorticoid vary in their genomic and non-genomic mechanism of action in A549 cells. Br J Pharmacol 2002; 135: 511-519.

20 Zeiner M, Gehring U. Glucocorticoid receptor expression during differentiation of human promyelocytic leukemia cells. Cancer Res 1993; 53: 3513-3517.

21 Shibakura M, Niiya K, Kiguchi $\mathrm{T}$, et al. Simultaneous induction of matrix metalloproteinase- 9 and interleukin 8 by all-trans retinoic acid in human PL-21 and NB4 myeloid leukaemia cells. Br J Haematol 2002; 118: 419-425.

22 Brown DC, Tsuji H, Larson RS. All-trans retinoic acid regulates adhesion mechanism and transmigration of the acute promyelocytic leukemia cell line NB-4 under physiologic flow. Br J Haematol 1999; 107: 86-98.

23 Marchetti M, Falanga A, Giovanelli S, Oldani E, Barbui T. All-trans retinoic acid increases adhesion to endothelium of the human promyelocytic leukemia cell line NB4. $\mathrm{Br}$ J Haematol 1996; 93: 360-366.

24 Zang C, Liu H, Ries C, Ismair MG, Petrides PE. Enhanced migration of the acute promyelocytic leukemia cell line NB4 under in vitro conditions during short-term all-transretinoic acid treatment. J Cancer Res Clin Oncol 2000; 126: 33-40.

25 Devy L, Hollender P, Munaut C, et al. Matrix and serine protease expression during leukemic cell differentiation induced by aclacinomycin and all-trans-retinoic acid. Biochem Pharmcol 2002; 63: 179-189.

26 Kuebler WM, Parthasarathi K, Wang PM, Bhattacharya J. A novel signaling mechanism between gas and blood compartments of the lung. J Clin Invest 2000; 105: 905-913.

27 Ye RD. Leukocyte inflammatory mediators and lung pahtophysiology: an update. Am J Physiol Lung Cell Mol Physiol 2004; 286: L461-L462.

28 Rosseau S, Selhorst J, Wiechmann K, et al. Monocyte migration through the alveolar epithelial barrier: adhesion molecule mechanisms and impact of chemokines. J Immunol 2000; 164: 427-435.

29 Ong DE, Chytil F. Changes in levels of cellular retinol- and retinoic-acid-binding proteins of liver and lung during perinatal development of rat. Proc Natl Acad Sci USA 1976; 73: 3976-3978. 